\title{
Post-synthesis control of Berry phase driven magnetotransport in $\mathrm{SrRuO}_{3}$ films
}

\author{
E. Skoropata $\odot,{ }^{1, *}$ A. R. Mazza $\odot,{ }^{1}$ A. Herklotz,${ }^{1,2}$ J. M. Ok, ${ }^{1}$ G. Eres,${ }^{1}$ M. Brahlek,,${ }^{1}$ T. R. Charlton $\odot,{ }^{3}$ \\ H. N. Lee, ${ }^{1}$ and T. Z. Ward (1, ${ }^{1,}$ \\ ${ }^{1}$ Materials Science and Technology Division, Oak Ridge National Laboratory, Oak Ridge, Tennessee 37830, USA \\ ${ }^{2}$ Institute of Physics, Martin-Luther-University Halle-Wittenberg, Halle 06120, Germany \\ ${ }^{3}$ Neutron Science Division, Oak Ridge National Laboratory, Oak Ridge, Tennessee 37830, USA
}

(Received 7 December 2020; accepted 28 January 2021; published 15 February 2021)

\begin{abstract}
Controlling electronic band structure is an important step toward harnessing quantum materials for practical uses. This is exemplified in the spin-polarized bands near the Fermi level of a ferromagnet where small variation to the Berry curvature can be used to control the intrinsic anomalous Hall effect (AHE). Since these pathways are highly sensitive to crystal structure, iterative post-synthesis manipulation of the underlying lattice can act as a fundamental probe and provide new opportunities for functionalization. In this work, depth-dependent magnetic inhomogeneity in a $4 d$ itinerant ferromagnet $\mathrm{SrRuO}_{3}$ film is controlled by applying low energy He ion irradiation. Combined magnetometry, polarized neutron reflectometry, and magnetotransport experiments demonstrate that the Berry phase and associated AHE can be continuously and iteratively modified post-synthesis. The findings of this work should be widely applicable to other quantum materials where crystal distortions and symmetry are tightly bound to band structure and resulting Berry phase effects.
\end{abstract}

DOI: 10.1103/PhysRevB.103.085121

\section{INTRODUCTION}

Manipulating magnetotransport properties that originate from topologically nontrivial characteristics of functional quantum materials can provide a foundation for future technology requiring fast energy efficient sensors and devices. For example, the intrinsic anomalous Hall effect (AHE) of conducting magnetic materials can be understood using the framework of band structure topology as it relates to modifying functionally relevant Berry phase curvature effects $[1,2]$. A consequence of the band-structure relationship is that the AHE is intimately connected to symmetry, spin-orbit coupling, and disorder. Large AHEs are also expected to originate from nontrivial nodal points in Weyl and three-dimenional (3D) Dirac semimetals [3,4]. More recently, in systems hosting chiral spin textures like skyrmions, an additional topological Hall effect (THE) with dramatic field-dependent anomalies results from an emergent electromagnetic field and Berry phase in real space originating from the interaction of charge carriers with the magnetic spin topology [5,6]. Disentangling the unconventional magnetotransport signatures from real- and momentum-space Berry phases potentially existing simultaneously in complex materials systems [7-17]

\footnotetext{
${ }^{*}$ Present address: Swiss Light Source, Paul Scherrer Institut, 5232 Villigen PSI, Switzerland.

${ }^{\dagger}$ wardtz@ornl.gov
}

Published by the American Physical Society under the terms of the Creative Commons Attribution 4.0 International license. Further distribution of this work must maintain attribution to the author(s) and the published article's title, journal citation, and DOI. remains a considerable challenge that hinders the precise control of electronic functionalities. Most importantly, by discovering new approaches to continuously alter the structure and symmetry of complex materials systems we can open new pathways to understand the origin of novel functional properties.

A model system that exemplifies the complexity of understanding unconventional magnetotransport is $\mathrm{SrRuO}_{3}$. Here, Berry phase physics manifests in magnetotransport of this $4 d$ itinerant ferromagnet as an unconventional temperaturedependent AHE attributed to topologically nontrivial $t_{2 g}$ band crossings near the Fermi level $[7,18,19]$. Difficulties in understanding and controlling the AHE responses are the result of the band structure being extremely sensitive to dimensional confinement, structural symmetry, and disorder [9,11-17,2023]. Experimental approaches aimed at exploiting these sensitivities have relied on substrate- or interface-induced strain; however, these methods have proved incapable of providing the minute structural distortion and symmetry modifications that can be expected to be required for functionalization [24].

In this work, we demonstrate the continuous control of the intrinsic AHE in bulk-like films of $20 \mathrm{~nm}$ thickness by modifying lattice symmetry and unit-cell volume with low-energy helium ion irradiation to apply uniaxial lattice expansion along the film's out-of-plane direction [25-28]. We also provide an example of a fully experimental measure of the inhomogeneous ferromagnetism and intrinsic AHE with a depth-dependent magnetic profile measured using polarized neutron reflectometry to model the "hump-like anomaly" mimicking the topological Hall effect of $\mathrm{SrRuO}_{3}$ that is fully consistent with the bulk-senstive magnetometry and magnetotransport behaviors. Our method to continuously tune magnetotransport resulting from the Berry phase mechanism 

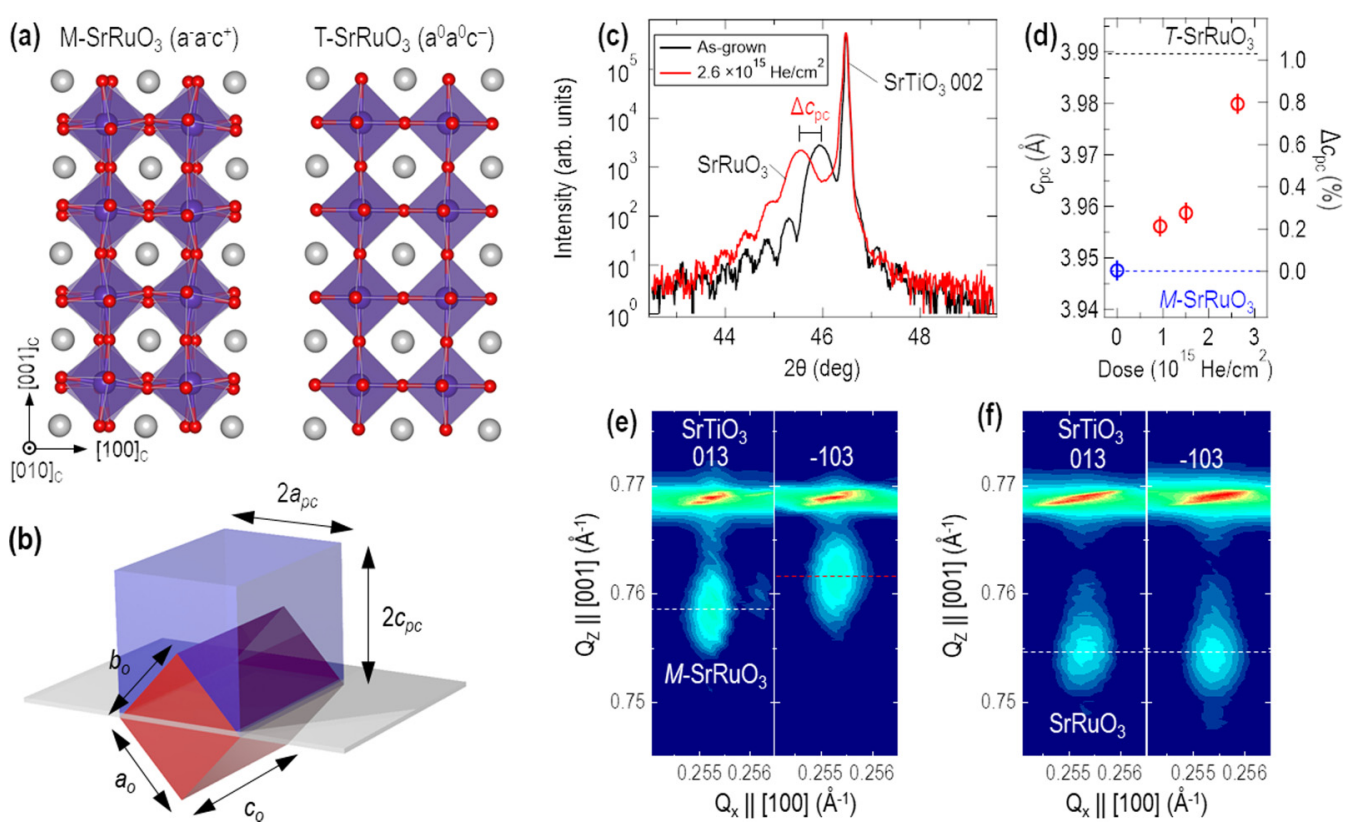

FIG. 1. (a) Illustration of the octahedral rotations of $\mathrm{SrRuO}_{3}$ in the orthorhombic $(M-\mathrm{SRO})$ and tetragonal ( $T$-SRO) phases. (b) Epitaxial relationship between the $M$-SRO and pseudocubic (pc) indices. (c) $\theta-2 \theta$ scans of the $\mathrm{SrRuO}_{3}$ thin films grown on $\mathrm{SrTiO}_{3}(001)$ substrates with different helium dosages. (d) The out-of-plane lattice parameter changes as a function of the helium dose labeled according to the pseudocubic unit cell as $c_{\mathrm{pc}}$. (e) Reciprocal space map around the (103) $)_{\mathrm{pc}}$ reflection of the as-grown sample is consistent with the typical orthorhombic phase, whereas (f) a nearly full transition to the undistorted tetragonal phase is indicated with the largest lattice expansion.

of $\mathrm{SrRuO}_{3}$ opens the possibility to control topological properties arising from the lattice strain that reaches far beyond the epitaxial interface. Our work points to an alternative synthetic approach to control the underlying symmetries that are closely linked to novel quantum phenomena.

\section{EXPERIMENTAL METHODS}

$\mathrm{SrRuO}_{3}$ films of $20 \mathrm{~nm}$ thickness are grown epitaxially on $\mathrm{SrTiO}_{3}$ (001) substrates using pulsed laser deposition followed by iterative low-energy helium ion irradiation to modify out-of-plane lattice expansion and subsequent octahedral rotations [28]. To design a desired strain profile through the film thicknesses Stopping and Range of Ion Motion (SRIM) models are used to guide a selection of sacrificial gold cap layer thickness and ion acceleration voltages [28]. The cap layers are removed prior to magnetic and transport characterizations.

All samples were synthesized by pulsed laser epitaxy with a substrate temperature, oxygen partial pressure, and laser fluence of $700{ }^{\circ} \mathrm{C}, 100 \mathrm{mTorr}$, and $1.0 \mathrm{~J} / \mathrm{cm}^{2}$, respectively on atomically flat $\mathrm{TiO}_{2}$-terminated (001) $\mathrm{SrTiO}_{3}$ substrates with a $\operatorname{KrF}$ eximer laser $(\lambda=248 \mathrm{~nm})$. The crystal structure, phase purity, and orientation of these films were confirmed by x-ray reflectivity and diffraction measurements using a Panalytical $X$ 'pert four-circle X-ray diffractometer. Samples were helium implanted using a SPECS IQE 11/35 ion source. The optimal conditions for controlling ion implantation cross sections in films of 20 to $30 \mathrm{~nm}$ can be achieved by varying a sacrificial gold cap layer of thicknesses ranging from 10 to $25 \mathrm{~nm}$ in concert with varying implantation energy between 4 and 5 $\mathrm{keV}$.

The electronic transport measurements were performed with a 9 T Quantum Design PPMS (physical properties mea- surement system). The electrical contacts were made with indium solder in a van der Pauw configuration, and the fielddependent data were antisymmetrized. The magnetization was determined with a 7 T Quantum Design MPMS3 (magnetic properties measurement system), after subtracting a linear background to correct for the diamagnetic response of the $\mathrm{SrTiO}_{3}$ substrate [29].

Polarized neutron reflectivity was measured using the Magnetism Reflectometer (BL4A) located at the Spallation Neutron Source at Oak Ridge National Laboratory. An applied magnetic field of $4.7 \mathrm{~T}$ was provided by a warm-bore cryomagnet with the field direction parallel to the projection of the scattering plane onto the sample's surface. The sample was held in helium exchange gas at $2 \mathrm{~K}$ by a recondensing helium cryostat capable of providing a stable sample temperature between 1.8 and $800 \mathrm{~K}$.

\section{RESULTS AND DISCUSSION}

Bulk and as-grown thin film $\mathrm{SrRuO}_{3}$ adopts the Pbnm space group that is described by the Glazer notation $a^{-} a^{-} c^{+}$. With helium irradiation, a transformation to the tetragonal phase is accompanied by the removal of the rotations about the two in-plane axes to $a^{0} a^{0} c^{-}$and an expansion of the lattice in the out-of-plane direction [28], as illustrated in Fig. 1. Xray diffraction and reciprocal space maps (RSMs) of $\mathrm{SrRuO}_{3}$ films demonstrate that even under the largest helium dose the film's quality and expansion along the out-of-plane direction can be controlled while maintaining epitaxy to the substrate. Consistent with previous studies, the RSMs about the (103) reflection indicate that the octahedral rotations present in the epitaxially strained orthorhombic phase of the as-grown film along the in-plane directions are gradually reduced as uniaxial 

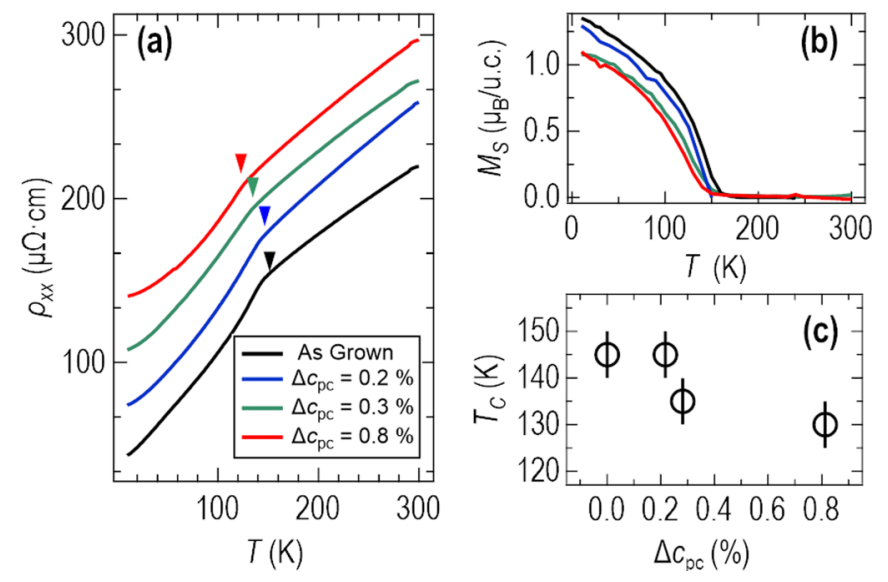

FIG. 2. (a) Resistivity $\rho_{\mathrm{xx}}$ and (b) saturation magnetization $M_{\mathrm{S}}$, are shown as a function of temperature, in addition to (c) the ferromagnetic Curie temperature $T_{\mathrm{C}}$ determined from $\rho_{\mathrm{xx}}(T)$.

out-of-plane expansion is applied [28,30,31]. The structural characterizations are used to quantify four different films with increasing lattice expansion. These states follow a relatively smooth transition from the as-grown orthorhombic state toward the most expanded out-of-plane lattice state which shows no orthorhombic distortions [28]. For simplicity, these states are described through the change in measured $c_{\mathrm{pc}}$-axis lattice length $\left(\Delta c_{\mathrm{pc}}\right)$ from the as-grown state (Fig. 1).

The as-grown $\mathrm{SrRuO}_{3}$ shows metallic behavior where the resistivity $\rho_{\mathrm{xx}}$ decreases with decreasing temperature. The prominent change in slope of $\rho_{\mathrm{xx}}(T)$ in Fig. 2(a) is due to a suppression of spin scattering on cooling to the ferromagnetic state [32] that was used to determine $T_{\mathrm{C}}=145 \mathrm{~K}$ for the as-grown $\mathrm{SrRuO}_{3}$ film. The saturation magnetization, $M_{\mathrm{S}}$, at $10 \mathrm{~K}$ of $1.5 \mu_{B} /$ unit cell, and $M_{S}(T)$ were consistent with the $T_{C}$ indicated by $\rho_{\mathrm{xx}}(T)$, and with the physical properties of other high quality epitaxial $\mathrm{SrRuO}_{3}$ films [33,34]. An increase in $\rho_{\mathrm{xx}}$ and decrease in $T_{\mathrm{C}}$ and $M_{\mathrm{S}}$ are observed with progressively larger lattice expansions. The observed change in $\rho_{\mathrm{xx}}$ and small reduction in $T_{\mathrm{C}}$ is in agreement with the transformation from the orthorhombic to the tetragonal phase as unit cell volume is increased [35].

To map how the structural transformation impacts the magnetotransport properties, the Hall resistivity $\rho_{\mathrm{xy}}$ is measured as a function of out-of-plane applied magnetic field $H_{\perp}$ from 10 to $150 \mathrm{~K}$ for different lattice expansions and is shown in Fig. 3. In a conventional ferromagnet, the Hall resistivity is described by the empirical relation $\rho_{\mathrm{xy}}=\rho_{\mathrm{OHE}}+\rho_{\mathrm{AHE}}=$ $R_{0} H_{\perp}+R_{\mathrm{S}} M_{\perp}$, where the ordinary Hall coefficient $\left(R_{0}\right)$ depends on the density of the charge carriers and the anomalous Hall resistivity follows the out-of-plane magnetization $\left(M_{\perp}\right)$ through the coefficient $\left(R_{S}\right)$ which depends on the longitudinal resistivity $\left(\rho_{\mathrm{xx}}\right)$. At the lowest temperatures, as-grown $\mathrm{SrRuO}_{3}$ exhibits $n$-type carriers, as characterized by a negative linear ordinary Hall effect $\rho_{\mathrm{OHE}}=R_{0} H$, where $R_{0}=1 / n e$ provides the carrier concentration $n=1.8 \times 10^{22} \mathrm{~cm}^{-3}$. The field dependence is characterized by a square-shaped $\rho_{\mathrm{AHE}}(H)$ with the coercive field $\left(H_{\mathrm{C}, \mathrm{AHE}}\right)$ required to reverse the sign of $\rho_{\mathrm{AHE}}(H)$ at a fixed temperature. The magnitude of $\rho_{\mathrm{AHE}}$ is defined as the magnitude of $\rho_{\mathrm{xy}}(H)$ well above the field-
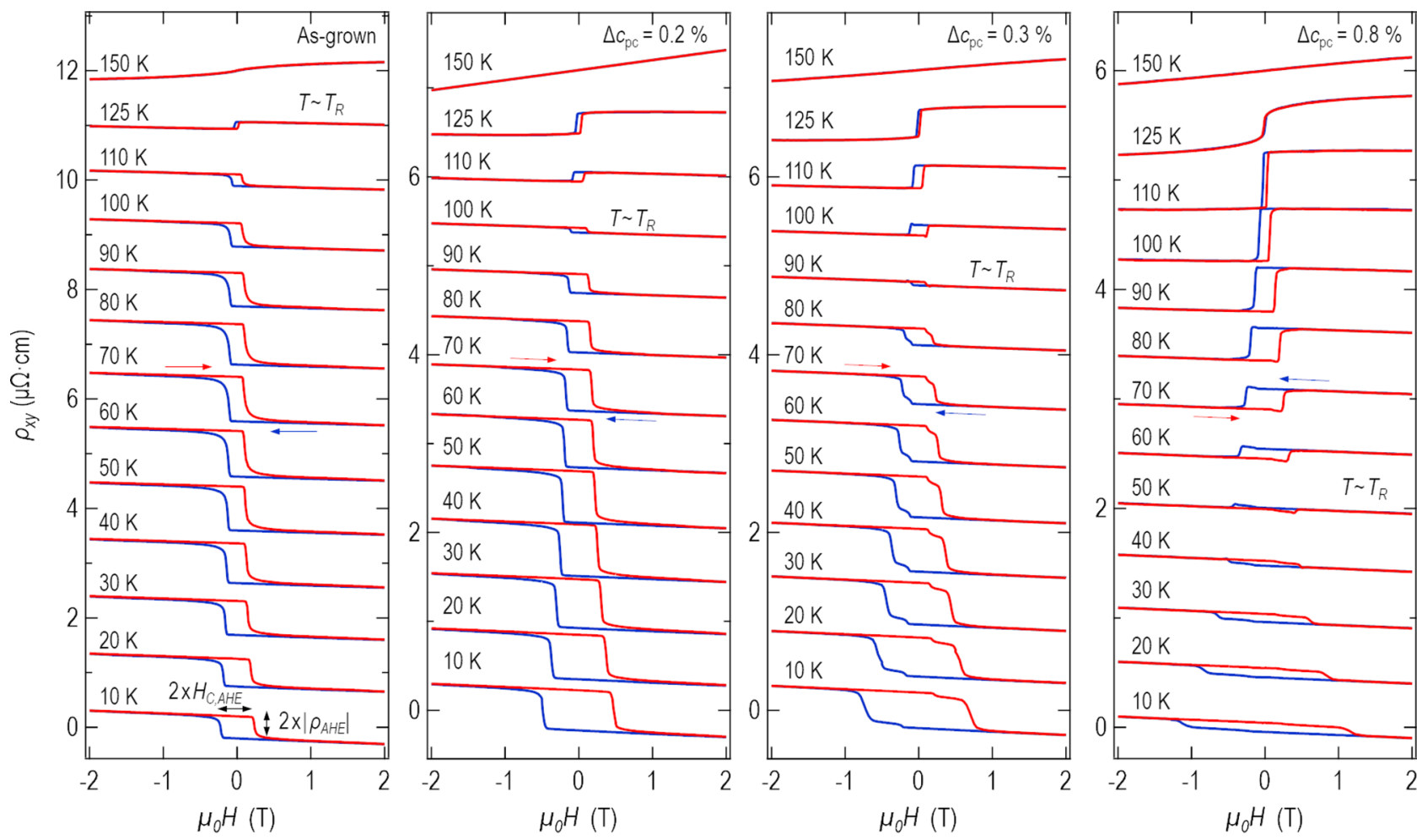

FIG. 3. Hall effect measurements of as-grown and helium irradiated $\mathrm{SrRuO}_{3}$ films. Definitions for the anomalous Hall resistivity $\rho_{\mathrm{AHE}}$ and coercive field $H_{\mathrm{C}, \mathrm{AHE}}$ are indicated at curves for the as-grown film measured at $10 \mathrm{~K}$. Note the scale differences labeled on the vertical axis, and curves at different temperatures are shown with a constant offset for clarity. 

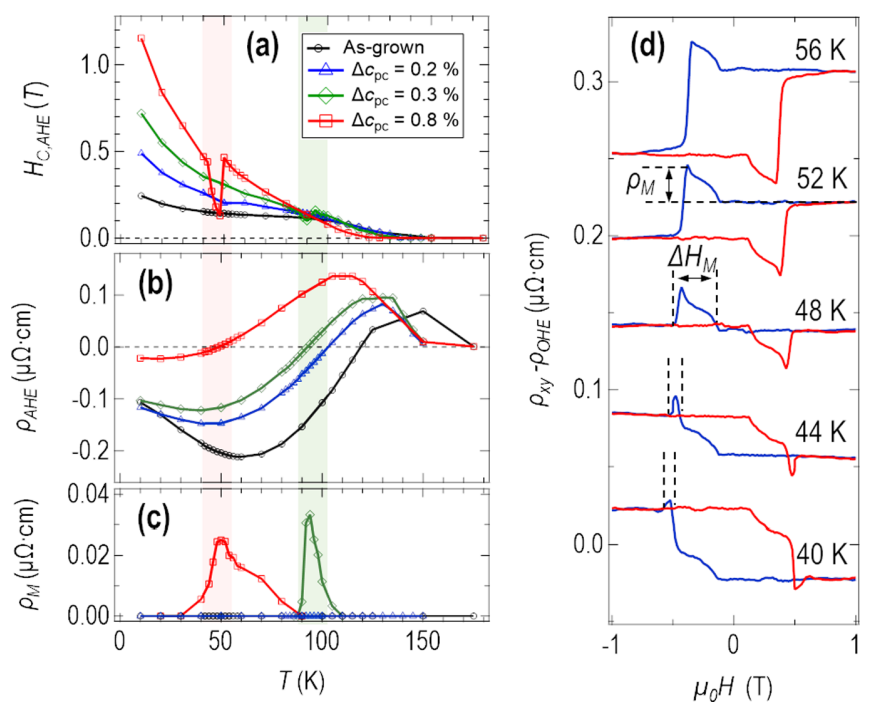

FIG. 4. The (a) zero crossing of the AHE with applied magnetic field, $H_{\mathrm{C}, \mathrm{AHE}}$, (b) $\rho_{\mathrm{AHE}}$, and (c) peak amplitude of the hump-like feature $\rho_{\mathrm{M}}$ are shown for as-grown and helium irradiated films. (d) Detailed temperature scans of the largest lattice expanded film for $T \sim T_{\mathrm{R}}$ showing the clear hump-like feature.

dependent hysteresis, after subtracting the linear contribution from $\rho_{\mathrm{OHE}}$. The observation that the anomalous Hall coefficient $R_{\mathrm{S}}$ has a negative value at low temperatures and changes sign at a reversal temperature $T_{\mathrm{R}} \sim 130 \mathrm{~K}$ is in agreement with previous reports of bulk and thin film $\mathrm{SrRuO}_{3}$ that show an unconventional $\rho_{\mathrm{AHE}}(T)$ due to $k$-space Berry phase effects [18].

It is evident that the magnetotransport properties summarized in Fig. 4 are all strongly influenced by structural modifications induced by helium irradiation. At $10 \mathrm{~K}$, the as-grown $\mathrm{SrRuO}_{3}$ shows a moderate $H_{\mathrm{C}, \mathrm{AHE}}$ typical of the approximate out-of-plane easy axis of the orthorhombic phase. A gradual but substantial increase in $H_{\mathrm{C}, \mathrm{AHE}}$ coincides with lattice expansion. This $H_{\mathrm{C}, \mathrm{AHE}}$ enhancement is consistent with a reorientation of the magnetic easy axis, which can be expected as lattice symmetry is shifted from an in-plane to out-of-plane tetragonality [36]. More intriguing, is the gradual tuning of the temperature dependence of $\rho_{\mathrm{AHE}}(T)$ and $T_{\mathrm{R}}$ that signifies a change in the Berry phase mechanism induced in a macroscopic and controlled manner as the lattice is expanded. Since the electronic transport occurs within the $\mathrm{RuO}_{6}$ network, changes in the unit cell volume or octahedral tilts and rotations are expected to modify the electronic properties due to the reduction of orbital overlap. Examining broadly the evolution of the Hall effect with uniaxial lattice expansion, there is a gradual tuning from the nearly all negative $\rho_{\mathrm{AHE}}$ behavior of the orthorhombic $\mathrm{SrRuO}_{3}$ towards the tetragonal phase that displays a positive $\rho_{\mathrm{AHE}}$ at all temperatures [37-40]. This is consistent with a scenario in which an increase in the unit cell volume modifies the Ru-O hybridization to tune states near the Fermi level.

At higher levels of lattice expansion, $\rho_{\mathrm{xy}}(H)$ also develops a complex shape including a prominent hump-like peak with $\left|\rho_{\mathrm{xy}}(H)\right|<\left|\rho_{\mathrm{AHE}}\right|$ around $T \sim T_{\mathrm{R}}$, as shown in Figs. 4(c) and 4(d). This additional Hall resistivity is defined as $\rho_{M}$ and
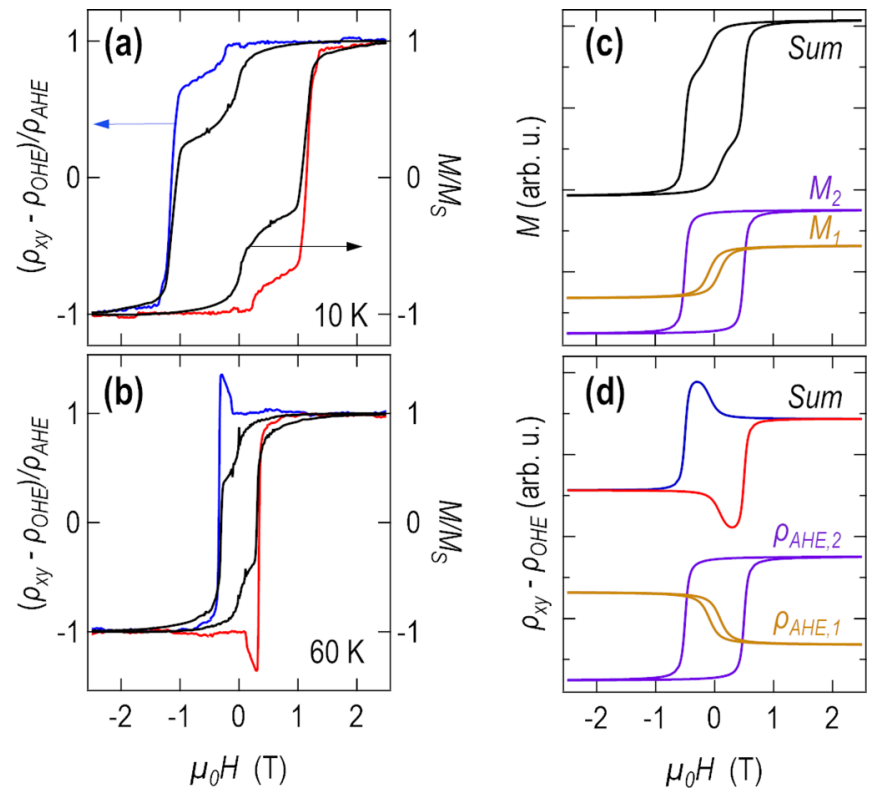

FIG. 5. A comparison of the normalized magnetization and anomalous Hall resistivity at (a) $10 \mathrm{~K}$ and (b) $60 \mathrm{~K}$ for helium irradiated $\mathrm{SrRuO}_{3}$ with the largest lattice expansion. Illustration of multicomponent field-dependent reversal of the (c) magnetization and (d) Hall effect resistivity for two arbitrary superimposed components with opposite sign anomalous Hall coefficient and different magnetic coercivities.

is quantified from the maximum deviation from $\rho_{\mathrm{AHE}}$. To understand this unconventional field-dependent response, it is essential to compare the $M(H)$ and $\rho_{\mathrm{xy}}(H)$ measured at the same temperatures to directly distinguish ferromagnetism from a potential noncollinear or chiral response. Although such a comparison is often overlooked in $\mathrm{SrRuO}_{3}$ ultrathin films and heterostructures displaying the unconventional magnetotransport properties, a lack of consideration of both the magnetization and magonetotransport response can lead to statements on mechanisms that are inconclusive. Figures 5(a) and 5(b) show clear feature overlaps between these measurements within regions of similar applied magnetic fields. Furthermore, the $H_{\mathrm{C}, \mathrm{AHE}}$ of $\rho_{\mathrm{xy}}$ displays an apparent nonmonotonic temperature dependence in the vicinity of $T_{\mathrm{R}}$ for films with a hump-like feature. This behavior indicates that multiple decoupled spin populations are present within the film and that each spin population possesses distinct field reversal behaviors. The occurrence of a hump-like anomaly has been observed recently in ultrathin $\mathrm{SrRuO}_{3}$ films and heterostructures, where inhomogeneities such as interfacial octahedral rotations are proposed to induce coexisting magnetic states within a $\mathrm{SrRuO}_{3}$ film $[20,37,40]$. The positive hump-like anomaly in helium irradiated $\mathrm{SrRuO}_{3}$ is quantitatively different from ultrathin $\mathrm{SrRuO}_{3}$ and heterostructures, which display a negative hump (see Supplemental Material (SM) Fig. 1 [41]). This suggests that the effects of helium-induced lattice expansion alter the intrinsic properties of $\mathrm{SrRuO}_{3}$ differently than natural epitaxial relaxation. Nevertheless, an interpretation based on inhomogeneous ferromagnetism, represented in the most simple case by two components with opposite AHE [Fig. 5(c)-5(d)], can produce 

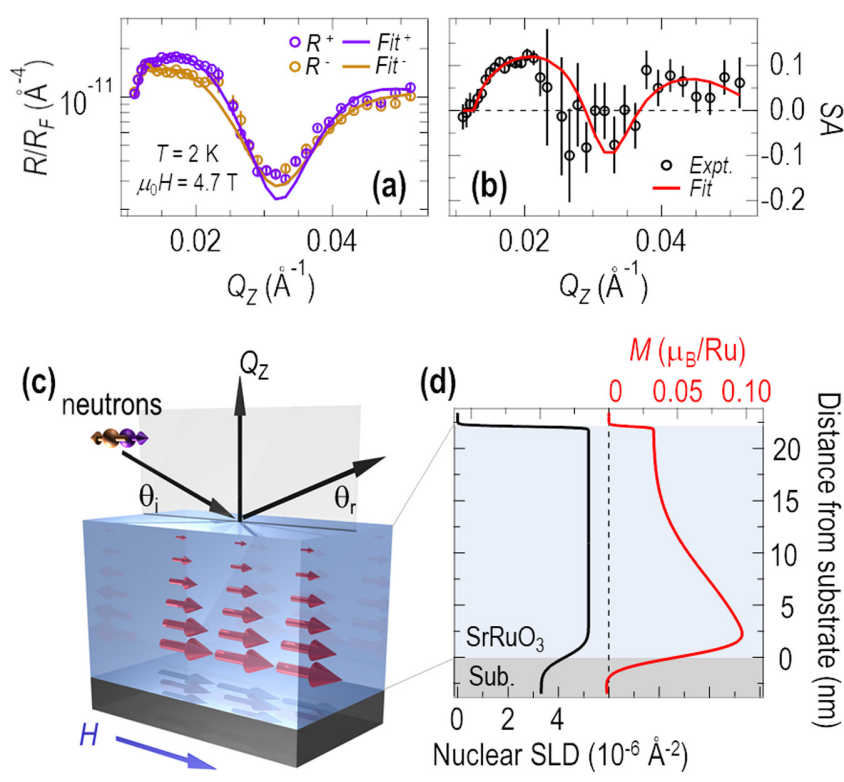

FIG. 6. Polarized neutron reflectometry (PNR) of helium irradiated $\mathrm{SrRuO}_{3}$. The experimental and fitted normalized (a) neutron reflectivity $\left(R / R_{\mathrm{F}}\right)$ and (b) spin asymmetry $(S A)$. (c) Illustration of the PNR experiment, and inhomogeneous magnetization profile of helium irradiated $\mathrm{SrRuO}_{3}$ film. (d) The best fit of the depthdependent nuclear density and magnetization profiles. Further detail of the fitting method used to obtain the result in (d) can be found in SM Sec. 3 [41].

the qualitative temperature-dependent change in $\rho_{\mathrm{xy}}(T)$ of helium irradiated $\mathrm{SrRuO}_{3}$.

While a model of the "hump-like anomaly" of $\mathrm{SrRuO}_{3}$ based on inhomogeneous ferromagnetism appears compelling, a complete understanding of the physical properties requires an understanding of the origin of the magnetic homogeneity. To investigate the magnetization profile to determine the film uniformity directly, polarized neutron reflectometry (PNR) measurements were performed on a helium irradiated film. In an effort to observe possible magnetic inhomogeneity, PNR measurements were conducted in a magnetic field of $4.7 \mathrm{~T}$ at $2 \mathrm{~K}$, well above fields showing magnetic hysteresis in Hall effect and magnetometry measurements. Fitting of the neutron and $\mathrm{x}$-ray reflectivity data was done using Parratt formalism [42] using the REFL1D software package [43]. Fits to the PNR data (normalized to $R_{\mathrm{F}}=$ asymptotic value of the Fresnel reflectivity [44] $16 \pi^{2} / Q^{4}$ ) for each spin state can be seen in Fig. 6(a). The resulting fit to the spin asymmetry, $S A=$ $\left[\left(R^{+}-R^{-}\right) /\left(R^{+}+R^{-}\right)\right]$, shown in Fig. 6(b) shows a gradient in the magnetization profile, as illustrated in Fig. 6(c) and plotted in Fig. 6(d). While a gradient in the magnetization is observed, the nuclear scattering length density, SLD, exhibits no inhomogeneity [Fig. 6(d)]. This result, sensitive to the atomic density of the film, suggests that the expansion is from He incorporation into the lattice rather than self-interstitials of $\mathrm{Sr}$ or $\mathrm{Ru}$. Three alternative models were considered for the spin asymmetry (SA) from PNR; one with a gradient allowing the maximum magnetization to be near the substrate, one with a gradient in the opposite direction, and one with uniform magnetization (shown in SM Fig. 2 [41]). The best fit to the experimental data is an inhomogeneous depth-dependent
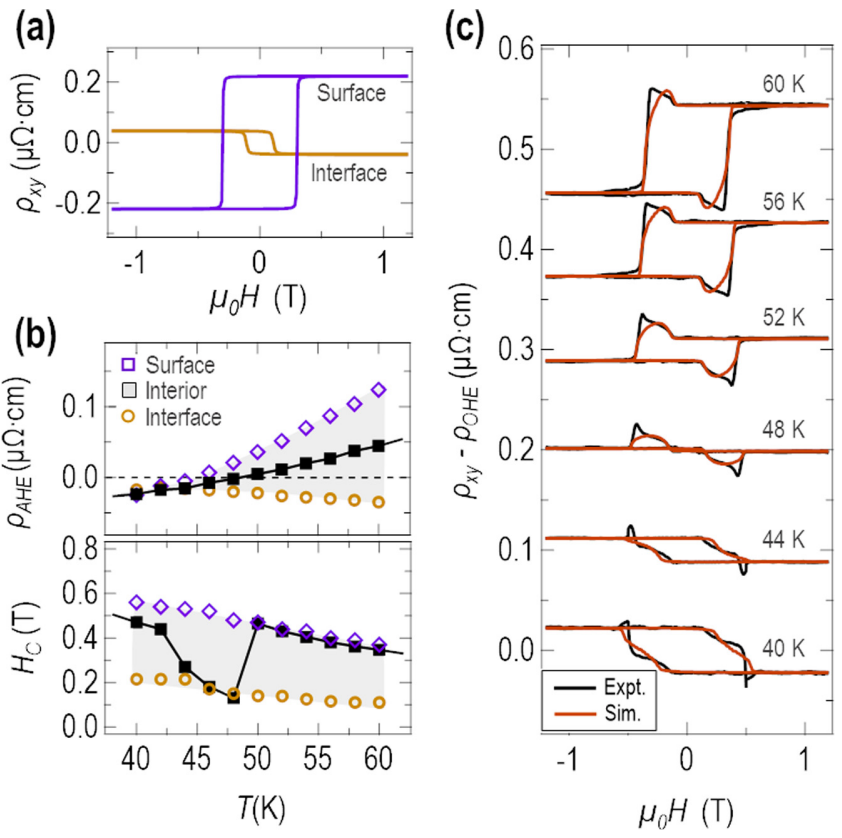

FIG. 7. Anomalous Hall effect sign reversal modeled using the depth-dependent magnetic profile obtained from neutron experiments. (a) $\rho_{x y}$ of the surface and interface components which define the limits of the profile distribution of the model (at $60 \mathrm{~K}$ ), and (b) shown for all temperatures where the black line indicates the film average identical to Figs. 4(a) and 4(b). (c) Comparison of the experimental Hall effect measurement for the $\mathrm{SrRuO}_{3}$ film with the largest $c_{\mathrm{pc}}$-axis expansion, and the model from the PNR-derived inhomogeneity profile, and distribution in $\rho_{\mathrm{xy}}$ and $H_{\mathrm{C}}$ from (b).

magnetization profile with the lowest magnetization at the surface of the film. Since it is known from magnetometry that an increasing helium dosage results in a decrease in $M_{\mathrm{S}}$ of $\mathrm{SrRuO}_{3}$, the PNR SA is consistent with a helium dose profile in which the largest influence resides near the surface and the lowest influence resides near the film-substrate interface, which is as expected from the SRIM profiles.

To fully describe the role of inhomogeneity on the magnetotransport properties of $\mathrm{SrRuO}_{3}$, we construct a model of the magnetization reversal and AHE using the magnetization profile obtained from PNR as a guide to the depth-dependent variation in intrinsic properties of the $\mathrm{SrRuO}_{3}$. We consider a film consisting of layers with distinct $\rho_{\mathrm{xy}, \mathrm{i}}(H, T)$, each described by different intrinsic $\rho_{\mathrm{AHE}, \mathrm{i}}(T)$ and $H_{\mathrm{C}, \mathrm{i}}(T)$ characteristics. A modified Langevin function is used to describe each component as $\rho_{\mathrm{xy}, \mathrm{i}}(T, H)=\rho_{\mathrm{AHE}, \mathrm{i}}(T)\left[\operatorname{coth}\left(\beta_{\mathrm{i}}\left(H \pm H_{\mathrm{C}, \mathrm{i}}(T)\right)-\right.\right.$ $\left.\left.1 /\left\{\beta_{\mathrm{i}}\left(H \pm H_{\mathrm{C}, \mathrm{i}}(T)\right)\right\}\right)\right]$, where $\beta_{\mathrm{i}}$ is a term that accounts for the sharpness of the magnetization reversal around $H_{\mathrm{C}}$ that depends on the details of the magnetic domain structure and density [45]. The depth-dependent values for $\rho_{\mathrm{AHE}, \mathrm{i}}(T)$, $H_{\mathrm{C}, \mathrm{i}}(T)$, and $\beta_{\mathrm{i}}$ were taken to vary with depth following the PNR profile shape of Fig. 6(d). The limiting values of the distribution of $\rho_{\mathrm{xy}, \mathrm{i}}$ and $H_{\mathrm{C}, \mathrm{i}}$ that represent the surface and interface are shown in Figs. 7(a) and 7(b). The total $\rho_{\mathrm{xy}}(T, H)$ of the film is modeled by choosing the limiting values of $\rho_{\mathrm{AHE}, \mathrm{i}}(T)$ and $H_{\mathrm{C}, \mathrm{i}}(T)$, and $\beta_{\mathrm{i}}$ of the surface and film-substrate interface, respectively, as shown in Figs. 7(a) and 7(b), and summed among 1-nm-thick increments of the 
film depth accounting for the parallel conduction of the layers [46] (additional details are in SM Fig. 3 [41]).

A comparison of the experimental and modeled $\rho_{\mathrm{xy}}(H, T)$ for the $\mathrm{SrRuO}_{3}$ with the largest $c_{\mathrm{pc}}$-axis lattice expansion is shown in Fig. 7(c). The depth-dependent gradient model of the AHE clearly reproduces the characteristic hump-like feature of $\mathrm{SrRuO}_{3}$ in the vicinity of $T_{\mathrm{R}}$. We note that since the PNR is sensitive only to the film's magnetization, is it possible that a strongly altered surface AHE (e.g., due to inversion symmetry breaking) may account for an additional surface component responsible for the small and sharp kink-like feature observed at the largest applied magnetic fields in Fig. 7(c). However, the multilayer magnetic model provides excellent reproduction of the experimentally observed temperature- and field-dependent AHE while being directly based on the real magnetization profile of the system. This points to the opportunity to predictively design real AHE devices.

These observations also directly address an ongoing topic of debate in the specific $\mathrm{SrRuO}_{3}$ system, where a two-layer or Gaussian inhomogeneity profile has been proposed recently in ultrathin $\mathrm{SrRuO}_{3}$ films, with a proposed origin of the multicomponents attributed to defects or vacancies, substrateinduced octahedral tilting, thickness effects, and inversion symmetry breaking $[15,16,23]$. The helium irradiation approach produces a depth-dependent inhomogeneity analogous to that observed in ultrathin films, although induced on a macroscopic scale, which can be controlled continuously with helium dosage profile. In addition, the AHE components of the He-irradiated $\mathrm{SrRuO}_{3}$ do not match those of the typical ultrathin $\mathrm{SrRuO}_{3}$, suggesting unique effects of the helium irradiation on the magnetic anisotropy and AHE when a high single-crystal quality is preserved. Finally, it is worth noting that, although a vertical spatial inhomogeneity has been proposed to provide the inversion symmetry breaking necessary for DMI [14], our observation of clear multiple decoupled spin populations present in both $\rho_{\mathrm{AHE}}(T)$ and in the $M(H)$ support multiple inhomogeneous ferromagnetic components and not a more complicated explanation of topological Hall effect. Furthermore, our description of the total Hall response from an experimentally derived spatial inhomogeneity profile obtained from neutron reflectometry experiments supports the recent theories that inhomogeneity and resulting multicomponent intrinsic AHE is responsible for the fielddependent hump-like anomalies [15-17,20,21,23] of ultrathin $\mathrm{SrRuO}_{3}$.
In conclusion, combined measurements of x-ray diffraction and polarized neutron reflectometry are used to map the change in crystal structure and magnetization profile through a film thickness. These cross sections are used to provide real space data for a magnetotransport model which shows that magnetic layering alone can generate the anomalous Hall effect observed in $\mathrm{SrRuO}_{3}$ films. We find that the systematic manipulation of lattice symmetry and local distortions possible with helium irradiation is found to be a highly effective means to tune magnetotransport properties driven by Berry phase effects in the prototypical $4 d$ itinerant ferromagnet $\mathrm{SrRuO}_{3}$ and that topological effects do not need to be considered to allow the model to predictively design AHE in this material. These findings enable future studies of Berry phase physics and electronic transport in any epitaxial system with nontrivial states near the Fermi level by providing a post-synthesis method to continuously and iteratively tune structural symmetry and local distortion profiles.

The Department of Energy will provide public access to these results of federally sponsored research in accordance with the DOE Public Access Plan [47].

\section{ACKNOWLEDGMENTS}

Experimental design, irradiation processing, and all characterization was supported by the U.S. Department of Energy (DOE), Basic Energy Sciences, Materials Sciences and Engineering Division. A portion of this research used resources at the Spallation Neutron Source and the Center for Nanophase Materials Science, a DOE Office of Science User Facility operated by the Oak Ridge National Laboratory. ORNL is managed by by UT-Battelle, LLC under Contract No. DE-AC05-00OR22725 with the U.S. Department of Energy. Sample synthesis performed by A.H. was supported by the federal state of Saxony-Anhalt and the "European Regional Development Fund" and the German Research Foundation (DFG) - Grant No. HE8737/1-1. The United States Government retains and the publisher, by accepting the article for publication, acknowledges that the United States Government retains a nonexclusive, paid-up, irrevocable, worldwide license to publish or reproduce the published form of this manuscript, or allow others to do so, for United States Government purposes.
[1] D. Xiao, M.-C. Chang, and Q. Niu, Berry phase effects on electronic properties, Rev. Mod. Phys. 82, 1959 (2010).

[2] N. Nagaosa, J. Sinova, S. Onoda, A. H. MacDonald, and N. P. Ong, Anomalous Hall effect, Rev. Mod. Phys. 82, 1539 (2010).

[3] N. P. Armitage, E. J. Mele, and A. Vishwanath, Weyl and Dirac semimetals in three-dimensional solids, Rev. Mod. Phys. 90, 015001 (2018).

[4] A. Bansil, H. Lin, and T. Das, Colloquium: Topological band theory, Rev. Mod. Phys. 88, 021004 (2016).

[5] A. Neubauer, C. Pfleiderer, B. Binz, A. Rosch, R. Ritz, P. G. Niklowitz, and P. Böni, Topological Hall Effect in the $A$ Phase of MnSi, Phys. Rev. Lett. 102, 186602 (2009).
[6] P. Bruno, V. K. Dugaev, and M. Taillefumier, Topological Hall Effect and Berry Phase in Magnetic Nanostructures, Phys. Rev. Lett. 93, 096806 (2004).

[7] L. Klein, J. Reiner, T. Geballe, M. Beasley, and A. Kapitulnik, Extraordinary Hall effect in $\mathrm{SrRuO}_{3}$, Physica B 281-282, 608 (2000).

[8] N. Haham, Y. Shperber, M. Schultz, N. Naftalis, E. Shimshoni, J. W. Reiner, and L. Klein, Scaling of the anomalous hall effect in $\mathrm{SrRuO}_{3}$, Phys. Rev. B 84, 174439 (2011).

[9] J. Matsuno, N. Ogawa, K. Yasuda, F. Kagawa, W. Koshibae, N. Nagaosa, Y. Tokura, and M. Kawasaki, Interface-driven 
topological Hall effect in $\mathrm{SrRuO}_{3}-\mathrm{SrIrO}_{3}$ bilayer, Sci. Adv. 2 , e1600304 (2016).

[10] K.-Y. Meng, A. S. Ahmed, M. Baćani, A.-O. Mandru, X. Zhao, N. Bagués, B. D. Esser, J. Flores, D. W. McComb, H. J. Hug, and F. Yang, Observation of nanoscale skyrmions in $\mathrm{SrIrO}_{3} / \mathrm{SrRuO}_{3}$ bilayers, Nano Lett. 19, 3169 (2019).

[11] Y. Gu, Y.-W. Wei, K. Xu, H. Zhang, F. Wang, F. Li, M. S. Saleem, C.-Z. Chang, J. Sun, C. Song, J. Feng, X. Zhong, W. Liu, Z. Zhang, J. Zhu, and F. Pan, Interfacial oxygenoctahedral-tilting-driven electrically tunable topological hall effect in ultrathin $\mathrm{SrRuO}_{3}$ films, J. Phys. D: Appl. Phys. 52, 404001 (2019).

[12] Y. Ohuchi, J. Matsuno, N. Ogawa, Y. Kozuka, M. Uchida, Y. Tokura, and M. Kawasaki, Electric-field control of anomalous and topological Hall effects in oxide bilayer thin films, Nat. Commun. 9, 213 (2018).

[13] L. Wang, Q. Feng, Y. Kim, R. Kim, K. H. Lee, S. D. Pollard, Y. J. Shin, H. Zhou, W. Peng, D. Lee, W. Meng, H. Yang, J. H. Han, M. Kim, Q. Lu, and T. W. Noh, Ferroelectrically tunable magnetic skyrmions in ultrathin oxide heterostructures, Nat. Mater. 17, 1087 (2018).

[14] C. Wang, C.-H. Chang, A. Herklotz, C. Chen, F. Ganss, U. Kentsch, D. Chen, X. Gao, Y.-J. Zeng, O. Hellwig, M. Helm, S. Gemming, Y.-H. Chu, and S. Zhou, Topological Hall effect in single thick $\mathrm{SrRuO}_{3}$ layers induced by defect engineering, Adv. Electron. Mater. 6, 2000184 (2020).

[15] D. J. Groenendijk, C. Autieri, T. C. van Thiel, W. Brzezicki, J. R. Hortensius, D. Afanasiev, N. Gauquelin, P. Barone, K. H. W. van den Bos, S. van Aert, J. Verbeeck, A. Filippetti, S. Picozzi, M. Cuoco, and A. D. Caviglia, Berry phase engineering at oxide interfaces, Phys. Rev. Research 2, 023404 (2020).

[16] D. Kan, T. Moriyama, K. Kobayashi, and Y. Shimakawa, Alternative to the topological interpretation of the transverse resistivity anomalies in $\mathrm{SrRuO}_{3}$, Phys. Rev. B 98, 180408(R) (2018)

[17] L. Wu, F. Wen, Y. Fu, J. H. Wilson, X. Liu, Y. Zhang, D. M. Vasiukov, M. S. Kareev, J. H. Pixley, and J. Chakhalian, Berry phase manipulation in ultrathin $\mathrm{SrRuO}_{3}$ films, Phys. Rev. B 102, 220406 (2020).

[18] Z. Fang, N. Nagaosa, K. S. Takahashi, A. Asamitsu, R. Mathieu, T. Ogasawara, H. Yamada, M. Kawasaki, Y. Tokura, and K. Terakura, The anomalous Hall effect and magnetic monopoles in momentum space, Science 302, 92 (2003).

[19] S. Itoh, Y. Endoh, T. Yokoo, S. Ibuka, J.-G. Park, Y. Kaneko, K. S. Takahashi, Y. Tokura, and N. Nagaosa, Weyl fermions and spin dynamics of metallic ferromagnet $\mathrm{SrRuO}_{3}$, Nat. Commun. 7, 11788 (2016).

[20] L. Wang, Q. Feng, H. G. Lee, E. K. Ko, Q. Lu, and T. W. Noh, Controllable thickness inhomogeneity and Berry curvature engineering of anomalous Hall effect in $\mathrm{SrRuO}_{3}$ ultrathin films, Nano Lett. 20, 2468 (2020).

[21] B. Sohn, E. Lee, W. Kyung, M. Kim, H. Ryu, J. S. Oh, D. Kim, J. K. Jung, B. Kim, M. Han, T. W. Noh, B.-J. Yang, and C. Kim, Sign-tunable anomalous Hall effect induced by symmetryprotected nodal structures in ferromagnetic perovskite oxide thin films, arXiv:1912.04757.

[22] M. Ziese, L. Jin, and I. Lindfors-Vrejoiu, Unconventional anomalous Hall effect driven by oxygen-octahedra-tailoring of the $\mathrm{SrRuO}_{3}$ structure, J. Phys.: Materials 2, 034008 (2019).
[23] D. Kan and Y. Shimakawa, Defect-induced anomalous transverse resistivity in an itinerant ferromagnetic oxide, Phys. Status Solidi B 255, 1800175 (2018).

[24] T. H. Kim, D. Puggioni, Y. Yuan, L. Xie, H. Zhou, N. Campbell, P. J. Ryan, Y. Choi, J.-W. Kim, J. R. Patzner, S. Ryu, J. P. Podkaminer, J. Irwin, Y. Ma, C. J. Fennie, M. S. Rzchowski, X. Q. Pan, V. Gopalan, J. M. Rondinelli, and C. B. Eom, Polar metals by geometric design, Nature 533, 68 (2016).

[25] A. Herklotz, S. F. Rus, N. Balke, C. Rouleau, E.-J. Guo, A. Huon, S. KC, R. Roth, X. Yang, C. Vaswani, J. Wang, P. P. Orth, M. S. Scheurer, and T. Z. Ward, Designing morphotropic phase composition in $\mathrm{BiFeO}_{3}$, Nano Lett. 19, 1033 (2019).

[26] A. Herklotz, S. F. Rus, C. Sohn, S. KC, V. R. Cooper, E.J. Guo, and T. Z. Ward, Optical response of $\mathrm{BiFeO}_{3}$ films subjected to uniaxial strain, Phys. Rev. Mater. 3, 094410 (2019).

[27] H. Guo, S. Dong, P. D. Rack, J. D. Budai, C. Beekman, Z. Gai, W. Siemons, C. M. Gonzalez, R. Timilsina, A. T. Wong, A. Herklotz, P. C. Snijders, E. Dagotto, and T. Z. Ward, Strain Doping: Reversible Single-Axis Control of a Complex Oxide Lattice via Helium Implantation, Phys. Rev. Lett. 114, 256801 (2015).

[28] A. Herklotz, A. T. Wong, T. Meyer, M. D. Biegalski, H. N. Lee, and T. Z. Ward, Controlling octahedral rotations in a perovskite via strain doping, Sci. Rep. 6, 26491 (2016).

[29] J. M. D. Coey, M. Venkatesan, and P. Stamenov, Surface magnetism of strontium titanate, J. Phys.: Condens. Matter 28, 485001 (2016).

[30] A. Vailionis, H. Boschker, W. Siemons, E. P. Houwman, D. H. A. Blank, G. Rijnders, and G. Koster, Misfit strain accommodation in epitaxial $\mathrm{ABO}_{3}$ perovskites: Lattice rotations and lattice modulations, Phys. Rev. B 83, 064101 (2011).

[31] W. Lu, P. Yang, W. D. Song, G. M. Chow, and J. S. Chen, Control of oxygen octahedral rotations and physical properties in $\mathrm{SrRuO}_{3}$ films, Phys. Rev. B 88, 214115 (2013).

[32] L. Klein, J. S. Dodge, C. H. Ahn, G. J. Snyder, T. H. Geballe, M. R. Beasley, and A. Kapitulnik, Anomalous Spin Scattering Effects in the Badly Metallic Itinerant Ferromagnet $\mathrm{SrRuO}_{3}$, Phys. Rev. Lett. 77, 2774 (1996).

[33] A. Kanbayasi, Magnetic properties of $\mathrm{SrRuO}_{3}$ single crystal, J. Phys. Soc. Jpn. 41, 1876 (1976).

[34] G. Cao, S. McCall, M. Shepard, J. E. Crow, and R. P. Guertin, Thermal, magnetic, and transport properties of singlecrystal $\mathrm{Sr}_{1-x} \mathrm{Ca}_{x} \mathrm{RuO}_{3}<\sim x<\sim 1.0$, Phys. Rev. B 56, 321 (1997).

[35] A. Vailionis, W. Siemons, and G. Koster, Room temperature epitaxial stabilization of a tetragonal phase in $A \mathrm{RuO}_{3}$ $(A=\mathrm{Ca}$ and Sr) thin films, Appl. Phys. Lett. 93, 051909 (2008).

[36] A. Herklotz, Z. Gai, Y. Sharma, A. Huon, S. F. Rus, L. Sun, J. Shen, P. D. Rack, and T. Z. Ward, Designing magnetic anisotropy through strain doping, Adv. Sci. 5, 1800356 (2018).

[37] F. Bern, M. Ziese, K. Dörr, A. Herklotz, and I. Vrejoiu, Hall effect of tetragonal and orthorhombic $\mathrm{SrRuO}_{3}$ films, Phys. Status Solidi R 7, 204 (2012).

[38] D. Kan, R. Aso, H. Kurata, and Y. Shimakawa, Unit-cell thick $\mathrm{BaTiO}_{3}$ blocks octahedral tilt propagation across oxide heterointerface, J. Appl. Phys. 115, 184304 (2014). 
[39] D. Kan, R. Aso, H. Kurata, and Y. Shimakawa, Epitaxial strain effect in tetragonal $\mathrm{SrRuO}_{3}$ thin films, J. Appl. Phys. 113, 173912 (2013).

[40] M. Ziese and I. Vrejoiu, Anomalous and planar Hall effect of orthorhombic and tetragonal $\mathrm{SrRuO}_{3}$ layers, Phys. Rev. B 84, 104413 (2011).

[41] See Supplemental Material at http://link.aps.org/supplemental/ 10.1103/PhysRevB.103.085121 for an illustration of the typical two-component "hump-like" anomaly of ultrathin $\mathrm{SrRuO}_{3}$, and additional details of the depth dependent ferromagnetic inhomogeneity model for polarized neutron reflectometry and Hall effect measurements.

[42] L. G. Parratt, Surface Studies of Solids by Total Reflection of X-Rays, Phys. Rev. 95, 359 (1954).
[43] P. Kienzle, B. Maranville, K. O’Donovan, J. Ankner, N. Berk, and C. Majkrzak, https://www.nist.gov/ncnr/reflectometrysoftware, 2017.

[44] S. Singh, M. R. Fitzsimmons, T. Lookman, J. D. Thompson, H. Jeen, A. Biswas, M. A. Roldan, and M. Varela, Magnetic Nonuniformity and Thermal Hysteresis of Magnetism in a Manganite Thin Film, Phys. Rev. Lett. 108, 077207 (2012).

[45] R. Harrison, A physical model of spin ferromagnetism, IEEE Trans. Magn. 39, 950 (2003).

[46] R. L. Petritz, Theory of an experiment for measuring the mobility and density of carriers in the space-charge region of a semiconductor surface, Phys. Rev. 110, 1254 (1958).

[47] U.S. DOE Public Access Plan, http://energy.gov/downloads/ doe-public-access-plan 